\title{
DSD: Nur indizierte medizinische Behandlungen in früher Kindheit
}

\section{Mirjam Werlen*}

Dr. iur., LL.M., Bern

\footnotetext{
Die Autorin ist Verfasserin der Dissertation «Persönlichkeitsschutz des Kindes, höchstpersönliche Rechte und Grenzen elterlicher Sorge im Rahmen medizinischer Praxis. Das Beispiel von Varianten der Geschlechtsentwicklung und DSD», Bern 2014, vgl. dort Glossar.
}

Yvon Heller und Françoise Narring ist zu danken für ihren Beitrag in der Schweizerischen Ärztezeitung [1], in dem sie über die KRK im Anschluss an den 3. Überprüfungszyklus der Schweiz durch den UN-Kinderrechtsausschuss im Januar 2015 berichten.

Die Kinderrechtskonvention (KRK) verpflichtet die Vertragsstaaten, die im Übereinkommen verankerten Rechte jedes Kindes zu achten, zu gewährleisten bzw. zu schützen sowie alle geeigneten Gesetzgebungs-, Verwaltungs- und sonstigen Massnahmen zu ergreifen [2]. Es müssen also gemäss Praxis und Lehre drei Verpflichtungsarten unterschieden werden: eine negative Unterlassungspflicht des Staates bzw. ein Abwehranspruch der Berechtigten gegen den Staat (duty to respect); eine positive - präventive oder kurative - Schutzpflicht des Staates, menschenrechtlich geschützte Rechtsgüter vor Gefahren oder Übergriffen auch durch Dritte zu schützen (duty to protect), und drittens, die Menschenrechte sicherzustellen und zu gewährleisten in der Gesetzgebung und Verwaltung, im Verfahren und im Alltag, z.B. die medizinische Versorgung betreffend (duty to ensure/fulfill) [3]. Von Bedeutung ist auch, dass justiziable Schutzpflichten dann entstehen, wenn der Staat Kenntnis von drohenden oder erfolg-

\section{Zusammenfassung}

Die Kinderrechtskonvention (KRK) verpflichtet die Vertragsstaaten, die im Übereinkommen verankerten Rechte jedes Kindes zu achten, zu gewährleisten und zu schützen. Staatliche Schutzpflichten entstehen dann, wenn der Staat Kenntnis von drohenden/erfolgten Gefahren erhalten hat oder davon wissen musste, was bei DSD («Disorders of Sex Development») der Fall ist.

Der Ausschuss für die Rechte des Kindes (CRC) überwacht u.a. die Einhaltung der KRK (kürzlich im Rahmen der Staatenberichte der Schweiz). Wesentlich ist der Schutz des Kindes vor Gewalt, aber auch, im Sinne der Ethikempfehlungen der NEK, dass bei DSD keine medizinisch nicht-indizierten Behandlungen in früher Kindheit vorgenommen werden. Der FMH bzw. der SAMW wird empfohlen, im Rahmen des nationalen Konzepts zu seltenen Krankheiten und zu sozialversicherungsrechtlichen Leistungen bzw. psychologischen Beratungs- und Unterstützungsangeboten der Eltern direkt nach der Geburt Richtlinien zu verfassen.

\section{DSD («Disorders of Sex Development»)}

DSD («Disorders of Sex Development», deutsch: "Störungen der Geschlechtsentwicklung»): 2005 wurde auf der Konsensuskonferenz von Chicago [8] als medizinische Bezeichnung für "Intersexualität»/«Intersex» der Terminus "Disorders of Sex Development» (DSD) vereinbart; dieser Begriff hat sich in der medizinischen Literatur durchgesetzt.

Vor dem Hintergrund, dass die Thematik der "Intersexualität» eine der körperlichen Geschlechtlichkeit ist und nicht eine der Sexualität, ist der Ausdruck «Intersexualität» abzulehnen, da er das Gegenteil suggeriert. Auch der Ausdruck DSD unterliegt der Kritik, da im Fall der Abwesenheit somatischer und psychischer Leiden dennoch als Störung bezeichnet wird, was als Variante der Geschlechtsentwicklung verstanden werden kann. Insbesondere wird der Begriff "Intersexualität» von Menschen mit Klinefelter-, Turner- und Adrenogenitalem Syndrom (AGS), die sich klar mit dem männlichen oder weiblichen Geschlecht identifizieren, abgelehnt. Ferner ist problematisch, dass der Ausdruck "Störung" an die medizinische Perspektive gebunden ist, so dass die lebensweltliche und psychosoziale in den Hintergrund tritt. Deshalb wird hier DSD als "Differences of Sex Development» im Sinne einer Variante der Geschlechtsentwicklung verstanden [9]. Vorzuziehen wäre also "Variations of Sex Development»/ «Varianten der Geschlechtsentwicklung" (VSD).

ten Gefahren und/oder Übergriffen erhalten hat oder bei pflichtgemässer Sorgfalt darum wissen musste; Letzteres ist bei DSD ("Disorders of Sex Development", Näheres siehe Kasten) der Fall.

\section{Sensibilisierungsarbeit verstärken}

Dem Ausschuss für die Rechte des Kindes (CRC) obliegt auch die Aufgabe, die Einhaltung der KRK zu überwachen, was er in seinen Schlussbemerkungen zum zweiten, dritten und vierten Staatenbericht der Schweiz getan hat [4]. Neben den von Yvon Heller und Françoise Narring festgehaltenen Punkten, und neben vom Ausschuss auch positiv beurteilten, in Kraft getretenen Gesetzen und institutionellen und politischen Massnahmen, hat er aber auch Bedenken angemeldet. So empfiehlt er insbesondere, die Sensibilisierungsarbeit zu verstärken (ein Beispiel wäre die von Yvon Heller und Françoise Narring erwähnte medizinische Ausbildung) und Schulungsprogramme zu den Kinderrech- 
ten für Berufsgruppen zu entwickeln (Ziff. 21) sowie v.a. das Grundprinzip des «best interest» (Art. 3 KRK) im Sinne der Konvention stärker auf allen staatlichen Ebenen zu verankern und der Öffentlichkeit bekannt zu machen (Ziff. 27), was im medizinischen Kontext auch die Achtung der Meinung des auch noch urteilsunfähigen Patienten betrifft (Art.12 KRK).

Was im Besonderen die Gewalt gegen Kinder betrifft, geht es auch um den Schutz des Kindes vor jeglicher Form von Gewalt (Ziff. 40f., es fehlen hier offenbar umfassende Daten und Studien zu Kindern) und um sogenannt schädliche Praktiken insbesondere bei DSD (Ziff. 42f.).

\section{DSD: Empfehlungen an die FMH/SAMW}

Der Ausschuss empfiehlt der Schweiz, im Sinne der Ethikempfehlungen der NEK [5] sicherzustellen, dass keine unnötigen medizinischen Behandlungen oder chirurgischen Eingriffe im Säuglingsalter oder in der Kindheit vorgenommen werden, dass die körperliche Unversehrtheit, die Autonomie und die Selbstbestimmung der betroffenen Kinder gewährleistet und geeignete Beratungs- und Unterstützungsangebote für $\mathrm{Fa}$ milien mit «intersexuellen» Kindern bereitgestellt werden (Ziff. 43). Der FMH bzw. der SAMW wird empfohlen, diesbezüglich Empfehlungen oder Richtlinien zu verfassen, wie sie dies auch zu anderen medizinischethisch-komplexen Fragestellungen getan hat.

Abgesehen von der für das schweizerische Recht erfolgten juristischen Analyse zur Einwilligungsreichweite der Eltern für ihr urteilsunfähiges Kind operative Genitalkorrekturen betreffend, soweit operative Eingriffe nicht medizinisch indiziert sind [6], stellt sich bei DSD in der Tat auch die Frage nach einer Unterstützung der Eltern direkt nach der Geburt - einerseits im Rahmen des nationalen Konzepts zu seltenen Krankheiten [7] und andererseits im Rahmen sozialversicherungsrechtlicher Leistungen bzw. psychologischer Unterstützung für Eltern und Kind. Denn eine grosse Zahl der DSD-Formen, wie etwa das AGS (Adrenogenitales
Syndrom und Nebenniereninsuffizienz), ist in der Verordnung über Geburtsgebrechen, also im invalidenversicherungsrechtlichen Zusammenhang, geregelt. Auch zu diesem letzten Punkt und zum Einbezug von DSD in das nationale Konzept zu seltenen Krankheiten ist die FMH bzw. die SAMW aufgerufen, Stellung zu beziehen.

Literatur

1 Heller Y, Narring F. Empfehlungen des UN-Kinderrechtsausschusses an die Schweiz. Schweiz Ärztezeitung 2016;97(16):604 Zusammenfassung aus Empfehlungen des UN-Kinderrechtsausschusses an die Schweiz, Bericht der Sensibilisierungstagung vom 17. September in Genf, Paediatrica (27) 2016/Spezialnummer Migranten, S. 23-5.

2 Siehe www.humanrights.ch zur KRK. Neben der KRK sind Fakultativprotokolle in Kraft, u.a. das dritte Fakultativprotokoll von 2011, welches es erlaubt, sich beim Ausschuss für die Rechte des Kindes (CRC) über die Verletzung einzelner Rechte der Konvention bzw. der beiden Fakultativprotokolle von $2000 \mathrm{zu}$ beklagen (Individualbeschwerdeverfahren). Letzteres gilt in absehbarer Zukunft auch für die Schweiz.

3 Für viele Naguib Tarek u.a., Diskriminierungsrecht, Bern 2014, N 121-4.

4 Comité des droits de l'enfant, Observations finales concernant les deuxième à quatrième rapports périodiques de la Suisse, soumis en un seul document, $\mathrm{CRC} / \mathrm{C} / \mathrm{CHE} / \mathrm{CO} / 2-4,26.2 .2015$, unter: www.ohchr.org $\rightarrow$ Sessions (Session No 68); es existiert eine hier wörtlich zitierte Übersetzung vom Bundesamt für Sozialversicherungen

5 Nationale Ethikkommission im Bereich Humanmedizin (NEK), Zum Umgang mit Varianten der Geschlechtsentwicklung. Ethische Fragen zur «Intersexualität», Stellungnahme Nr. 20/2012, Bern November 2012, unter: www.nek-cne.ch.

6 Werlen M. Persönlichkeitsschutz des Kindes, höchstpersönliche Rechte und Grenzen elterlicher Sorge im Rahmen medizinischer Praxis. Das Beispiel von Varianten der Geschlechtsentwicklung und DSD, Bern 2014 (Dissertation).

7 Dazu BAG, Nationales Konzept Seltene Krankheiten, Bern 2014 unter: www.bag.admin.ch $\rightarrow$ Themen $\rightarrow$ Krankheiten und Medizin, mit weiteren Informationen.

8 Hughes Ieuan A, Houk C, Faisal AS, Lee PA. Consensus statement on management of intersex disorders, Statement of the Lawson Wilkins Pediatric Endocrine Society (LWPES)/European Society for Paediatric Endocrinology (ESPE) Consensus Group, Journal of Pediatric Urology 2006/3, S. 148-162; oder Holterhus Paul-Martin, Grundlagen und Klinik der Störungen der Geschlechtsentwicklung, Monatsschrift Kinderheilkunde - Zeitschrift für Kinder- und Jugendmedizin 2008/3, S. 217-25.

9 Vgl. Stellungnahme der Bundesärztekammer «Versorgung von Kindern, Jugendlichen und Erwachsenen mit Varianten/ Störungen der Geschlechtsentwicklung (Disorders of Sex Development, DSD)», 30. Januar 2015, Deutsches Ärzteblatt 2015 $(112) / 13$, S. $1-12$ 